\title{
Entanglement Hamiltonian of interacting systems: Local temperature approximation and beyond
}

\author{
Mahdieh Pourjafarabadi $\odot,{ }^{1, *}$ Hanieh Najafzadeh $\odot,{ }^{1, *}$ Mohammad-Sadegh Vaezi, ${ }^{2}$ and Abolhassan Vaezi $\oplus^{1, \dagger}$ \\ ${ }^{1}$ Department of Physics, Sharif University of Technology, Tehran 14588-89694, Iran \\ ${ }^{2}$ Pasargad Institute for Advanced Innovative Solutions (PIAIS), Tehran 19916-33361, Iran
}

(Received 2 December 2020; revised 9 February 2021; accepted 11 February 2021; published 8 March 2021)

\begin{abstract}
We investigate the second quantization form of the entanglement Hamiltonian $(\mathrm{EH})$ of various subregions for the ground state of several interacting lattice fermions and spin models. The relation between the EH and the model Hamiltonian itself is an unsolved problem for the ground state of generic local Hamiltonians. In this paper, we demonstrate that the EH is practically local and its dominant components are related to the terms present in the model Hamiltonian up to a smooth spatially varying temperature even for (a) discrete lattice systems, (b) systems with no emergent conformal or Lorentz symmetry, and (c) subsystems with nonflat boundaries, up to relatively strong interactions. We show that the mentioned local temperature at a given point decays in a manner inversely proportional to its distance from the boundary between the subsystem and the environment. We find the subdominant terms in the $\mathrm{EH}$ as well and show that they are severely suppressed away from the boundaries of subsystem and are relatively small near them.
\end{abstract}

DOI: 10.1103/PhysRevResearch.3.013217

Introduction. Entanglement is a unique feature of quantum mechanics and serves as an essential tool in quantum information, quantum gravity, identification of topological order, quantum phase transition, etc. [1,1-18]. The entanglement Hamiltonian $(\mathrm{EH})$ associated with a subregion $A$ embedded in a manifold $M=A \cup B$ is defined as $\rho_{A}=e^{-K_{A}}$. Here, $\rho_{A}=\operatorname{Tr}_{B} \rho_{M}$ denotes the reduced density matrix (RDM) of $A$, where $\rho_{M}$ represents the total density matrix. One important question that arises from this definition is the relation between $K_{A}$ and $H_{A}$, the Hamiltonian terms with support only in region $A$. In fact, this problem dates back to the nineteenth century. A cornerstone of the classical statistical mechanics is that a subsystem $A$ at thermal equilibrium with its environment $(B)$ is described by a thermal ensemble with $K_{A}=H_{A} / T_{0}\left(k_{B}=\right.$ $\hbar=c=1$ ), where $T_{0}$ is a uniform and position-independent temperature. Furthermore, the eigenstate thermalization hypothesis conjectures that the RDM of highly excited quantum states will look thermal, again with $K_{A}=H_{A} / T_{0}$, where the uniform temperature $T_{0}$ in this case is dictated by the energy density [19]. In this paper, we revisit this fundamental problem, and using the density-matrix-renormalization-group (DMRG) approach, we obtain the second quantization form of $K_{A}$ for a number of interacting model Hamiltonians and for a variety of boundary shapes and conditions. Comparing the components of $K_{A}$ and $H_{A}$, we demonstrate that the above-mentioned statements are not quite accurate and for the ground state of local Hamiltonians, $K_{A}$ is indeed well

\footnotetext{
*These authors contributed equally to this work.

${ }^{\dagger}$ Corresponding author: vaezi@ sharif.edu
}

Published by the American Physical Society under the terms of the Creative Commons Attribution 4.0 International license. Further distribution of this work must maintain attribution to the author(s) and the published article's title, journal citation, and DOI. approximated by a local and nonuniform temperature rather than a uniform one.

The theoretical form of $K_{A}$ is known only for a limited class of continuum models with conformal symmetry (or Lorentz symmetry at zero temperature) and only for certain geometries of $A$ (e.g., half-space or ball geometry). It is known that under these conditions, (i) $K_{A}$ is local, (ii) the $\mathrm{EH}$ density is related to the Hamiltonian density via a smooth local temperature, namely, $K_{A}=\int_{x \in A} d^{d} x \mathcal{K}_{A}(x)=\int_{x \in A} d^{d} x \frac{\mathcal{H}(x)}{T(x)}$, and (iii) $T(x)$ approaches $T_{0}$, the equilibrium temperature of the entire system, far away from $\partial A$ (the boundary of $A$ ) and grows as $\frac{v}{2 \pi r(x)}$ at distance $r$ near $\partial A$. Here, $v$ is the group velocity of low-energy excitations [1,20-28]. We refer to these findings as the local temperature approximation (LTA) [26,27].

The LTA can be justified using the following intuitive argument. In thermal systems, the entropy density is proportional to their temperatures. On the other hand, for ground states, instead of the thermal entropy, we deal with the entanglement entropy, which is not precisely an extensive property. Nevertheless, we can still consider and gauge the contribution of individual degrees of freedom residing inside $A$ to the overall entanglement entropy between $A$ and $B, S_{A}$. Indeed, quantum mutual information can be one candidate to quantify such local contributions. Due to the decay of quantum mutual information with distance for the ground state of local Hamiltonians, the degrees of freedom that live near $\partial A$ are more entangled with those residing at $B$ than more distant ones. Accordingly, we can assign an effective quantum local temperature to different subregions of $A$ proportional to their contributions to $S_{A}$, which as just discussed must diminish away from $\partial A$.

Numerically, the EH of free fermions and free bosons can be evaluated easily [29-31]. However, for interacting models, it becomes highly nontrivial and challenging. Recently, several studies have analyzed the $\mathrm{EH}$ of quasi-one-dimensional conformal invariant or integrable models [32-48]. 
Nonetheless, we are still lacking a systematic derivation of the EH for larger and generic interacting systems and for various boundary geometries. In this paper, we address this problem and introduce a DMRG-based algorithm that enables us to extract the $\mathrm{EH}$ for a broader spectrum of problems.

$J_{1}-J_{2}$ Heisenberg model. Let us first discuss the form of $K_{A}$ for the $J_{1}-J_{2}$ Heisenberg model $\left(J_{1}=1\right)$ with the following Hamiltonian on the square lattice:

$$
H=J_{1} \sum_{\langle\mathbf{i}\rangle \in M} \mathbf{S}_{\mathbf{i}} \cdot \mathbf{S}_{\mathbf{j}}+J_{2} \sum_{\langle\langle\mathbf{i j}\rangle\rangle \in M} \mathbf{S}_{\mathbf{i}} \cdot \mathbf{S}_{\mathbf{j}} .
$$

The above Hamiltonian respects a SU(2) symmetry. Hence $K_{A}$ must respect $\mathrm{SU}(2)$ symmetry as well and thus is expanded as follows:

$$
K_{A}=\sum_{\mathbf{i}, \mathbf{j} \in \mathbf{A}} g_{J, \mathbf{i j}} \mathbf{S}_{\mathbf{i}} \cdot \mathbf{S}_{\mathbf{j}}+\cdots .
$$

Note that there is no restriction on $\mathbf{i}=\left(i_{x}, i_{y}\right)$ and $\mathbf{j}=\left(j_{x}, j_{y}\right)$ except that both must belong to $A$. In this paper, we have dropped higher-order terms since the retained terms already yield satisfactory results.

The DMRG technique is based on identifying the most relevant basis states of the Hilbert space [49]. Then we truncate the Hilbert space and discard the less relevant states. The number of kept states, which controls the accuracy of the DMRG, is called the bond dimension $\chi$, and its default value equals $2^{10}$ throughout this paper. The procedure of finding the truncation operators consecutively involves the computation and diagonalization of the RDM at every step of the DMRG and for different subsystem sizes. Hence $\rho_{A}$ is a natural by-product of the DMRG method and is available at every step. Moreover, every operator component of $K_{A}$ (e.g., $\mathbf{S}_{\mathbf{i}} \cdot \mathbf{S}_{\mathbf{j}}$ ) has a matrix representation in the DMRG, albeit in the truncated subspace. The remaining task is to adjust the EH's couplings, $g_{J, \mathbf{i j}}$, to bring our (simplified) guess for $K_{A}$, which we will denote as $\widetilde{K_{A}}$, close enough to the matrix representation of the RDM in the truncated Hilbert space achieved via the DMRG: $K_{A}=-\ln \rho_{A}$. To this end, we need to define an appropriate cost function as a measure of the distance between $\widetilde{K}_{A}$ and $K_{A}$. In our investigations, we mainly utilized the Hilbert-Schmidt distance between the Green's functions, namely, $\Delta_{1}:=\operatorname{Tr}_{A}\left(G_{A}-\widetilde{G}_{A}\right)^{2}$, where $G_{A, \mathbf{i j}}=\operatorname{Tr}_{A}\left(\mathbf{S}_{\mathbf{i}} \cdot \mathbf{S}_{\mathbf{j}} \rho_{A}\right)$ is the Green's function matrix achieved by the DMRG for $A$ and $\widetilde{G_{A}}$ denotes its counterpart evaluated using the trial $\mathrm{RDM}, \widetilde{\rho}_{A}=\exp \left(-\widetilde{K_{A}}\right)$ [50]. This cost function yields more reliable and reasonably robust results (against changing $\chi$ ) in the truncated Hilbert space than other candidates, e.g., the quantum relative entropy between $\rho_{A}$ and $\widetilde{\rho_{A}}$. We find the latter to overfit to numerical noises, e.g., the truncation and the computer's round-off errors (see Sec. 4 of the Appendix for more details). In the optimization procedure, we initialized $g_{J, \mathbf{i j}}$ based on general expectations from the LTA, e.g., the locality of $g_{J, \mathbf{i j}}$ and its linear dependence on $x_{\mathbf{i j}}$ (the minimum distance between $\overline{\mathbf{i j}}=\frac{\mathbf{i}+\mathbf{j}}{2}$ and $\partial A$ ). Then, we employed the gradient descent algorithm and let the cost function decide the optimum choice for $g_{J, \mathrm{ij}}$ (see the Appendix for more details).

We first focus on the $J_{2}=0$ Heisenberg model, which is unfrustrated and is known to host a Néel order on the square lattice [51,52]. Thus its ground state is a symmetry-broken
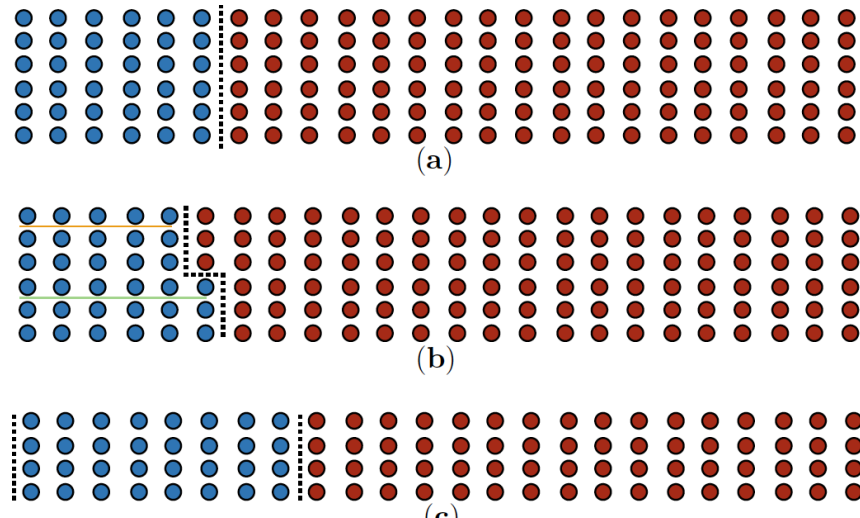

(c)

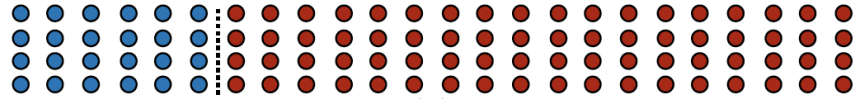
(d)

FIG. 1. (a)-(d) Various geometries of system and subsystem that we consider in this paper for computing the EH. The blue (red) sites define the subsystem $A(B)$. The dotted lines indicate the boundary between $A$ and $B, \partial A$. In (c), $\partial A$ comprises two disjoint surfaces since we have considered torus geometry. The green and orange lines in (b) illustrate the rows at which the couplings in Figs. 3(a) and 3(b) and in Figs. 3(c) and 3(d) are plotted, respectively.

phase with gapless Goldstone modes and does not respect the full conformal symmetry e.g., the translational and (around the center of plaquettes) rotational symmetries are broken. For this model, the system is always subject to the periodic boundary condition (PBC) along the $y$ axis.

(i) As the first example, we study the manifold and subsystem $A$ depicted in Fig. 1(a), where an open boundary condition (OBC) is imposed along $x$. In this case, $\partial A$ is flat, and its locus is given by $x_{b}=6+1 / 2$, the line which splits columns 6 and 7. The optimum couplings, $g_{J, \mathbf{i j}}$, which reproduce the DMRG's Green's functions (with less than $0.1 \%$ error), are plotted in Fig. 2.

Figure 2(a) shows the nearest-neighbor (NN) couplings along $x$ and $y$ (more precisely, $\beta_{J, x}\left(i_{x}+1 / 2\right):=g_{J, \mathbf{i}, \mathbf{i}+\hat{\mathbf{x}}}$ and $\left.\beta_{J, y}\left(i_{x}\right):=g_{J, \mathbf{i}, \mathbf{i}+\hat{\mathbf{y}}}\right)$ which are independent of $i_{y}$ due to the $y$-axis translation preserving the shape of $A$. Indeed, $\beta_{J}$ 's are

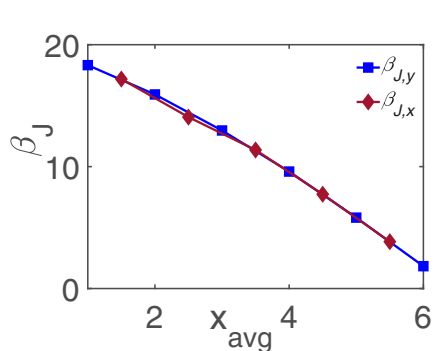

(a)

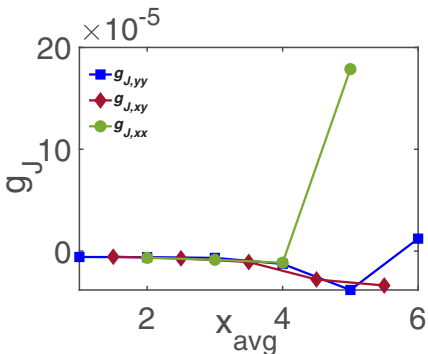

(b)
FIG. 2. EH's couplings for the Heisenberg model $\left(J_{2}=0\right)$ for the subsystem geometry shown in Fig. 1(a). (a) The inverse temperature profiles $\beta_{J, a}\left(x_{\mathrm{avg}}\right)=J_{1}^{-1} g_{J, a}(\mathbf{i}, \mathbf{j})(a=x, y)$ for the NN couplings vs the midpoint argument $x_{\mathrm{avg}}:=\frac{1}{2}\left(i_{x}+j_{x}\right)$. (b) Second- and thirdneighbor couplings vs $x_{\text {avg }}$. 


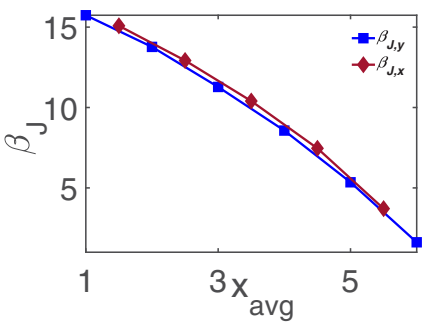

(a)

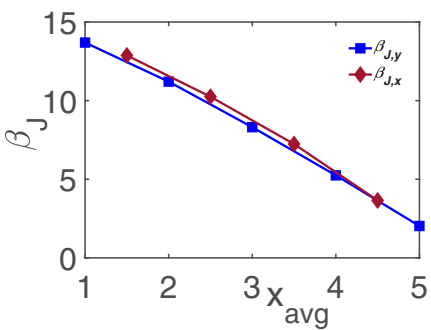

(c)

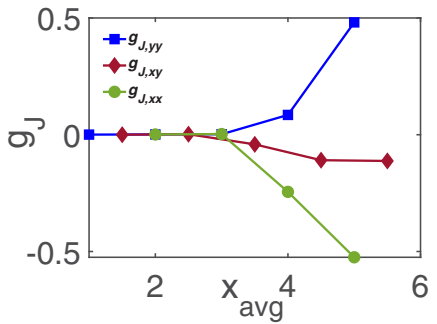

(b)

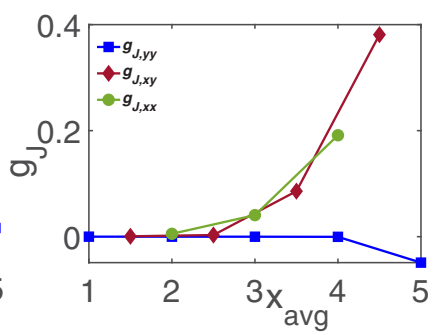

(d)
FIG. 3. EH's couplings for the Heisenberg model $\left(J_{2}=0\right)$ for the subsystem geometry shown in Fig. 1(b). Since $A$ breaks the translational symmetry along $y, g_{J,(\mathbf{i}, \mathbf{j})}$ depends on both $i_{x}$ and $j_{y}$. In (a) and (b), the couplings along the green line in Fig. 1(b) are plotted, and in (c) and (d), those corresponding to the orange line in Fig. 1(b) are plotted. The terms beyond the LTA grow substantially near $\partial A$ for nonflat boundary geometries compared with flat boundaries (cf. Fig. 2).

the inverse local temperature profiles. As Fig. 2(a) suggests, $\beta_{J, x}$ and $\beta_{J, y}$ follow the same profile, albeit if we shift the argument of $\beta_{x}$ by half of the lattice spacing. This shift is due to the fact that for $g_{J, x}$, the start and end points are located at different positions along $x$, while for $g_{J, y}$, the two points have identical $x$ values. In Sec. 4 of the Appendix, we demonstrate the robustness of $\beta_{J, x}$ and $\beta_{J, y}$ versus $\chi$. In Fig. 2(b), we have plotted $g_{J, x y}\left(i_{x}+1 / 2\right):=g_{J, \mathbf{i}, \mathbf{i}+\hat{\mathbf{x}}+\hat{\mathbf{y}}}, g_{J, y y}\left(i_{x}\right):=g_{J, \mathbf{i}, \mathbf{i}+2 \hat{\mathbf{y}}}$, and $g_{J, x x}\left(i_{x}+1\right):=g_{J, \mathbf{i}, \mathbf{i}+\mathbf{2} \hat{\mathbf{x}}}$. Their values are negligible everywhere, and they all die off quickly away from $\partial A$. These imply the locality of $K_{A}$ for the Heisenberg model when $\partial A$ is flat.

(ii) We now consider the same conditions as above, but this time with a curved $\partial A$ as shown in Fig. 1(b). In this case, $\beta_{J, x}$ and $\beta_{J, y}$ will depend on both $i_{x}$ and $i_{y}$. In Fig. 3, we have plotted $\beta_{J, x}$ and $\beta_{J, y}$ for the two different rows marked by orange and green lines in Fig. 1(b). Interestingly, the $\beta_{J, x}$ and $\beta_{J, y}$ profiles display a somewhat smooth curve satisfying our expectations from the LTA. The position dependence of the inverse temperature profile is more complicated in this problem, since the distance between $\overline{\mathbf{i j}}=(\mathbf{i}+\mathbf{j}) / \mathbf{2}$ and $\partial A$ depends on both its $x$ and $y$ components. In Figs. 3(b) and 3 (d) the second- and third-neighbor couplings are plotted for the above-mentioned rows. We see that for curved boundaries between $A$ and $B, K_{A}$ remains local everywhere, except close to $\partial A$, where we observe additional terms, though they are relatively small and subdominant.

(iii) Now, we consider the geometry illustrated in Fig. 1(c). Since the PBC is imposed on $M$ along both $x$ and $y$ directions, we have chosen $N_{y}=4$ to ensure $\chi=2^{10}$ is sufficient for the DMRG's convergence. As we see in Fig. 1(c), $\partial A$ is described by two surfaces: One of them separates columns 6 and 7 ,

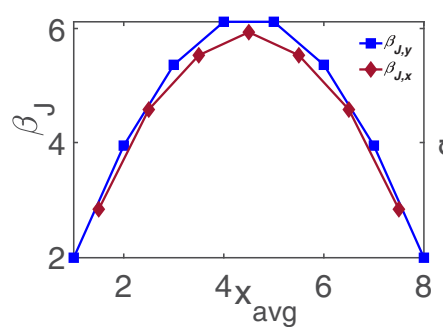

(a)

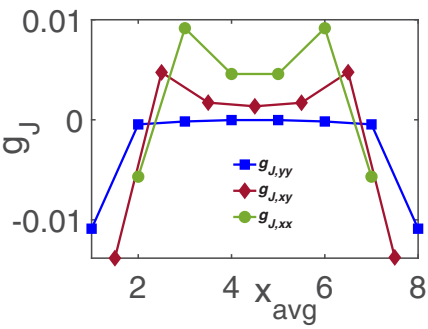

(b)
FIG. 4. (a) and (b) EH's couplings for the Heisenberg model $\left(J_{2}=0\right)$ subject to the PBC along $x$, for the subsystem geometry shown in Fig. 1(c). The NN couplings follow a parabolic curve and die off near both boundaries.

and the other one lies between the first and last columns. As a result, the LTA predicts that $\beta_{J, x}$ and $\beta_{J, y}$ must follow a parabolic form and vanish near both boundary surfaces. Figure 4 shows our numerical results for the nearest- as well as farther-neighbor couplings, both consistent with the LTA.

(iv) Let us now turn to the frustrated Heisenberg model with $J_{2}=0.6$, whose true ground state is not well understood, though it is conjectured to be a spin liquid phase with no classical spin order and with algebraically decaying spin-spin correlations [53]. For this model, we consider the geometry depicted in Fig. 1(d). The ground state is expected to be more entangled when $J_{2} / J_{1} \sim O(1)$. Hence we consider $N_{y}=4$ (again $N_{x}=24$ ) to ensure that the ground state is achieved reliably via $\chi=2^{10}$ in the DMRG. Since the Hamiltonian contains next-nearest-neighbor (NNN) couplings, we expect significant values for the NNN in $g_{J, \mathbf{i j}}$ as well. In Fig. 5(a), $\beta_{J, x}, \beta_{J, y}$, and also $\beta_{J, x y}\left(i_{x}+1 / 2\right):=\frac{1}{J} g_{J, \mathbf{i}, \mathbf{i}+\hat{\mathbf{x}}+\hat{\mathbf{y}}}$ are plotted. Again, we see that all these $\beta_{J}$ 's follow the same curve. Furthermore, Fig. 5(b) verifies the locality of $K_{A}$ everywhere except at $\partial A$, where $g_{J, y y}$ is about $29 \%(28 \%)$ of $g_{J, y}\left(g_{J, x y}\right)$ at that location.

Hubbard model. Here, we discuss the second quantization form of $K_{A}$ for the Hubbard model on the square lattice, whose Hamiltonian is

$$
\begin{aligned}
H= & -t_{1} \sum_{\langle\mathbf{i} \mathbf{j}\rangle \in M, \sigma} c_{\mathbf{i}, \sigma}^{\dagger} c_{\mathbf{j}, \sigma}-\mu \sum_{\mathbf{i} \in M, \sigma} n_{\mathbf{i}, \sigma} \\
& +U \sum_{\mathbf{i} \in M}\left(n_{\mathbf{i}, \uparrow}-\frac{1}{2}\right)\left(n_{\mathbf{i}, \downarrow}-\frac{1}{2}\right),
\end{aligned}
$$

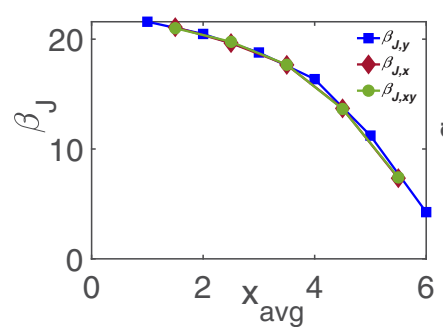

(a)

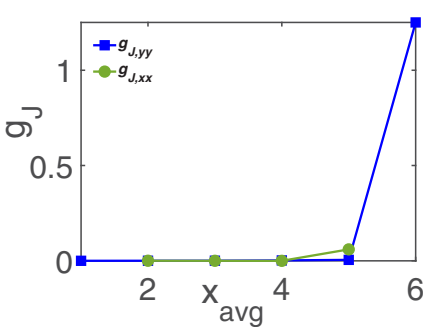

(b)
FIG. 5. (a) and (b) EH's couplings for the $J_{1}-J_{2}$ Heisenberg model $\left(J_{2}=0.6\right)$ for the subsystem geometry shown in Fig. 1(d). The LTA is valid everywhere, except at the boundary where the second-neighbor coupling along $y$ is non-negligible. 


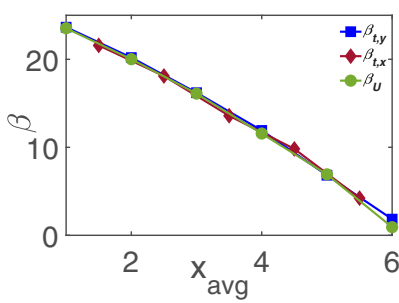

(a)

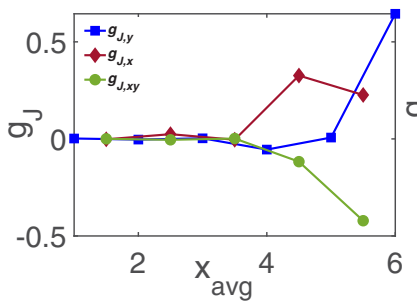

(c)

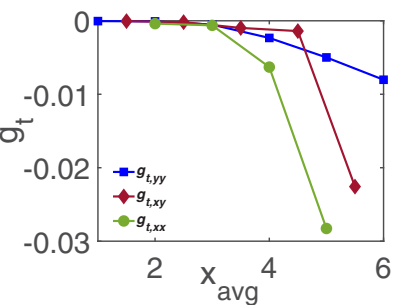

(b)

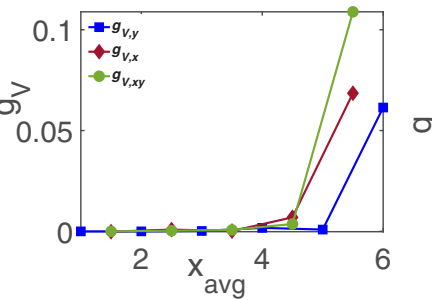

(d)
FIG. 6. (a)-(d) EH's couplings for the Hubbard model at half filling $\left(U=4 t_{1}\right)$ for the subsystem geometry shown in Fig. 1(d). LTA corrections are small (compared with the leading terms) everywhere, particularly away from $\partial A$. The particle-hole symmetry dictates that the couplings in (b) vanish. However, due to the finite truncation error of the DMRG at $\chi=2^{10}$, we obtain nonzero, though negligible, values.

where $n_{\mathbf{i}, \sigma}=c_{\mathbf{i}, \sigma}^{\dagger} c_{\mathbf{i}, \sigma}$. In this paper, we consider $t_{1}=1$, and $U=4$. The above Hamiltonian enjoys a $\mathrm{U}(1) \times \mathrm{SU}(2)$ symmetry for generic fillings. Accordingly, $K_{A}$ must be expanded as follows:

$$
\begin{aligned}
K_{A}= & -\sum_{\mathbf{i} \neq \mathbf{j} \in A} g_{t, \mathbf{i j}} c_{\mathbf{i}, \sigma}^{\dagger} c_{\mathbf{j}, \sigma}-\sum_{\mathbf{i} \in A, \sigma} g_{\mu, \mathbf{i}} n_{\mathbf{i}, \sigma} \\
& +\sum_{\mathbf{i} \in A} g_{U, \mathbf{i}}\left(n_{\mathbf{i}, \uparrow}-\frac{1}{2}\right)\left(n_{\mathbf{i}, \downarrow}-\frac{1}{2}\right) \\
& +\sum_{\mathbf{i} \neq \mathbf{j} \in A}\left[g_{V, \mathbf{i j}}\left(n_{\mathbf{i}}-1\right)\left(n_{\mathbf{j}}-1\right)+g_{J, \mathbf{i j}} \mathbf{S}_{\mathbf{i}} \cdot \mathbf{S}_{\mathbf{j}}\right]+\cdots,
\end{aligned}
$$

where $n_{\mathbf{i}}=n_{\mathbf{i}, \uparrow}+n_{\mathbf{i}, \downarrow}$ denotes the total electron number on site $\mathbf{i}$ and $S_{\mathbf{i}}^{x, y, z}=\sum_{a b} \frac{1}{2} c_{\mathbf{i}, a}^{\dagger} \sigma_{a, b}^{x, y, z} c_{\mathbf{i}, b}$ denotes the three components of the spin operator at $\mathbf{i}$. Again, we have discarded higher-order terms as we attain satisfactory results with the above structure. In the following, we consider both doped and undoped Hubbard models. In this section, we consider the geometry shown in Fig. 1(d).

(i) Let us start with the half-filling case. The ground state on the square lattice is described by a Néel antiferromagnetic spin order, and the charge or Mott gap opens up at moderate values of $U[54,55]$. Figure 6 summarizes our results for the optimum couplings of the EH. Here, motivated by the LTA, we define the following inverse temperatures: $\beta_{t, x}\left(i_{x}+1 / 2\right):=$ $g_{t, \mathbf{i}, \mathbf{i}+\hat{\mathbf{x}}}, \beta_{t, y}\left(i_{x}\right):=g_{t, \mathbf{i}, \mathbf{i}+\hat{\mathbf{y}}}$, and $\beta_{U}\left(i_{x}\right):=\frac{1}{U} g_{U, \mathbf{i}}$. According to Fig. 6, they all fairly follow an identical curve, albeit by considering the previously discussed $1 / 2$ shift in the argument of $\beta_{t, x}$. In Figs. 6(b)-6(d), we have shown the terms beyond the LTA, and again the locality of couplings is confirmed. Only near $\partial A$ are the additional terms non-negligible. Although $g_{V, \mathbf{i j}}$

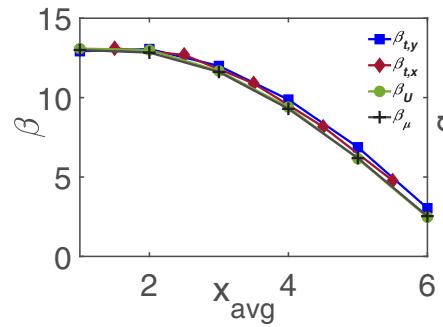

(a)

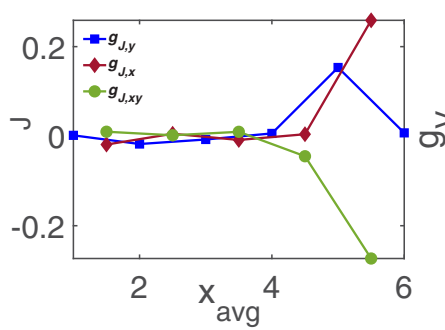

(c)

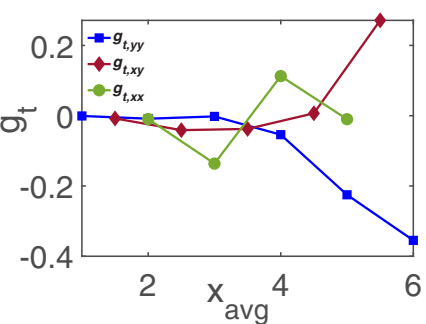

(b)

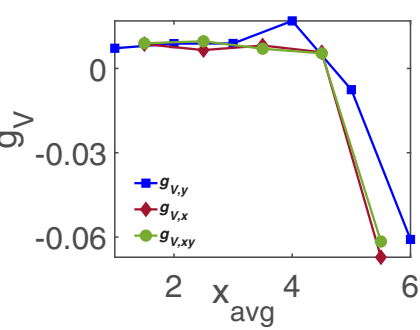

(d)
FIG. 7. (a)-(d) EH's couplings for the Hubbard model at the $p=1 / 8$ doping level $\left(U=4 t_{1}, \mu \approx-0.92 t_{1}\right)$ for the subsystem geometry shown in Fig. 1(d).

is insignificant everywhere, $g_{J, \mathbf{i j}}$ has decent values near $\partial A$; yet they are inferior to those of $g_{t,\langle\mathbf{i j}\rangle}$ and $g_{U, \mathbf{i}}$.

(ii) We now study the Hubbard model at $p=1 / 8$ doping, which is expected to have a stripe order and some tendency towards superconductivity [56-59]. The system is not expected to exhibit the Lorentz invariance or conformal symmetry for these symmetry-breaking phases. At finite doping, we need to define $\beta_{\mu}\left(i_{x}\right):=\frac{1}{\mu} g_{\mu, \mathbf{i}}$ as well (for the current example, $\mu \approx-0.92 t_{1}$ ). As Fig. 7 implies, various $\beta$ 's follow the expected trend, and the locality of couplings is again verified, albeit with growing corrections close to $\partial A$.

Summary. Our DMRG-based algorithm allowed us to access the second quantization form of the $\mathrm{EH}$ for several models and subsystem shapes. We showed that the $\mathrm{EH}$ is local and its dominant components are related to those of the Hamiltonian itself (more specifically, the stress-energy tensor) up to a single smooth local (inverse) temperature profile and confirmed the LTA. We studied the terms beyond the LTA and demonstrated that they are infinitesimal far away from $\partial A$ and relatively small near it. In the Appendix, we have provided more evidence which further corroborates our main findings. To our knowledge, the validity of the LTA for the ground state of local Hamiltonians for generic models that do not satisfy conformal algebra or even those with conformal symmetry but nonflat $\partial A$ is an unsolved problem despite active research. Our results suggest that the LTA is perhaps a legitimate assumption and applicable to a broader class of problems.

Our findings pave the way for several applications of the LTA. For instance, it can be shown that the LTA can practically solve the long-standing sign problem in quantum Monte Carlo methods and enable us to extract the ground-state properties of some unsolved interacting models. Furthermore, the LTA can be employed to enhance the performance and increase the accuracy of the DMRG technique. It can also be used to recover the entire spectrum and eigenstates of an unknown 
Hamiltonian by having access to its reduced density matrix (or correlation functions) associated with a rather small subregion of that system [28].

Acknowledgments. We gratefully acknowledge helpful discussions with M. Dalmonte, A. Lucas, S. Nezami, Z. Nussinov, H. Yarloo, A. Shahbazi, E. Huang, M. Kargarian, A. Rezakhani, and S. Alipour. A.V. acknowledges the Gordon and Betty Moore Foundations EPiQS Initiative through Grant No. GBMF4302 and Stanford Center for Topological Quantum Physics for partial financial support and hospitality during the completion of this work. M.-S.V. acknowledges the financial support from Pasargad Institute for Advanced Innovative Solutions (PIAIS) under a supporting grant scheme (Project No. SG1-RCM2001-01).

\section{APPENDIX}

In this Appendix, we will delve into the details of our algorithm and discuss the advantages and disadvantages of a number of cost function candidates along with their pairwise comparison, and we will present more results on the entanglement Hamiltonian (EH).

\section{Entanglement Hamiltonian in the truncated Hilbert space}

In general, the $\mathrm{EH}$ associated with subsystem $A$ which is defined as $K_{A}:=-\ln \rho_{A}$, can be expanded in terms of a complete basis of operators (not necessarily local) as follows (the tensor product of Pauli matrices, $\sigma_{a}, a=x, y, z$ with $\sigma_{0}=\mathbb{1}$, can generate a basis for all possible operators):

$$
K_{A}=\sum_{\alpha} g_{\alpha} \hat{O}_{\alpha}
$$

If we are given the reduced density matrix $\rho_{A}$, we can compute its logarithm (which requires a lot of consideration and special care when performed numerically) and achieve $K_{A}$ (up to the computer's round-off error). Having $K_{A}$ available, we can easily find the expansion coefficients $g_{\alpha}$ via the following relation:

$$
\vec{g}=M^{-1} \vec{J},
$$

where

$$
J_{\beta}=\operatorname{Tr}_{A}\left(K_{A} \hat{O}_{\beta}^{\dagger}\right), \quad M_{\alpha \beta}=\operatorname{Tr}_{A}\left(\hat{O}_{\alpha} \hat{O}_{\beta}^{\dagger}\right) .
$$

In the exact diagonalization (ED) method, $M_{\alpha, \beta} \propto \delta_{\alpha, \beta}$. Therefore $g_{\alpha} \propto \operatorname{Tr}_{A}\left(K_{A} \hat{O}_{\alpha}^{\dagger}\right)$. Consequently, we do not need to consider other operators if we are interested in reading the coefficient for a specific $\alpha$.

On the other hand, in the density-matrix-renormalizationgroup (DMRG) algorithm, instead of $\rho_{A}$ and $\hat{O}_{\alpha}$, we have to deal with $\overline{\rho_{A}}$ and $\overline{\hat{O}_{A}}$, which are their counterparts in the truncated Hilbert space and are defined as

$$
\overline{\rho_{A}}=T_{A}^{\dagger} \rho_{A} T_{A},
$$

and similarly for other operators, where $T_{A}$ denotes the truncation (also known as projection) operator. As before, we define $\overline{K_{A}}=-\ln \overline{\rho_{A}}$. Because of the numerous truncations involved in the DMRG, the situation is now more complicated for a few reasons: (i) The matrix $M$ is not diagonal, nor is it sparse. Due to consecutive truncations inherent to the DMRG method, most of operators have nonvanishing overlaps. Therefore we must consider all possible operators, including highly nonlocal ones such as string or brane operators. (ii) $M$ can be singular and have zero eigenvalues. As a result, it might not be invertible. (iii) The above method applied to the DMRG is very sensitive to various sources of numerical noise and error, such as the truncation, as well as the round-off error.

Besides the possibility of singular $M$, another main difficulty of applying the above algorithm to the DMRG is the annoying part which requires taking all possible operators into consideration. Below, we easily demonstrate that if $K_{A}$ contains a few relevant and dominant terms, then $\overline{K_{A}}$ contains exactly the same couplings and structure. To this end, recall that $T_{A}$ is achieved upon concatenating the dominant eigenvectors of $\rho_{A}$. Therefore

$$
\overline{\rho_{A}}=T_{A}^{\dagger} \rho_{A} T_{A}=T_{A}^{\dagger} e^{-K_{A}} T_{A}=e^{-T_{A}^{\dagger} K_{A} T_{A}} .
$$

Therefore

$$
\overline{K_{A}}:=-\ln \overline{\rho_{A}}=T_{A}^{\dagger} K_{A} T_{A} .
$$

Accordingly,

$$
\overline{K_{A}}=\sum_{\alpha} g_{\alpha} T_{A}^{\dagger} \hat{O}_{\alpha} T_{A}=\sum_{\alpha} g_{\alpha} \overline{\hat{O}_{\alpha}}
$$

hence, assuming $\overline{K_{A}}=\sum_{\alpha} \overline{g_{\alpha}} \overline{\hat{O}_{\alpha}}$,

$$
\overline{g_{\alpha}}=g_{\alpha} \text {. }
$$

\section{Algorithm and cost function}

In the above-mentioned method, for ED, we can ignore insignificant couplings since $M$ is diagonal. Nevertheless, when we apply this method to the DMRG, we have to retain all terms, no matter how infinitesimal they are, due to the complex form of $M$. Therefore we must come up with a better algorithm to find $g_{\alpha}$ without having to consider all irrelevant terms. For that purpose, we must consider a valid cost function. From our physical intuitions and expectations, we can think of the following three choices (as of now, we drop the overline sign and keep in mind that all operators are defined in the truncated Hilbert space):

(i) Hilbert-Schmidt distance between the Green's functions (GFs): $\Delta_{1} \equiv \operatorname{Tr}\left(G_{A}-\widetilde{G_{A}}\right)^{2}$, where $G_{A}(\alpha, \beta)=$ $\operatorname{Tr}_{A}\left(O_{\alpha}^{\dagger} O_{\beta} \rho_{A}\right)$ and $\widetilde{G_{A}}(\alpha, \beta)=\operatorname{Tr}_{A}\left(O_{\alpha}^{\dagger} O_{\beta} \widetilde{\rho_{A}}\right)$. Here, $\rho_{A}$ denotes the reduced density matrix (RDM) achieved via the DMRG for the desired subsystem, and $\widetilde{\rho_{A}}$ denotes the one achieved by combining the basis operators $\left(O_{\alpha}\right)$ with $g_{\alpha}$ coefficients that are yet to be determined. The basis of this method is that the RDM contains all the information about the equal time correlation functions within the subsystem. Thus, if we find a RDM which recovers all the correlation functions correctly, it must be identical to the actual one.

We would like to emphasize that in the DMRG, due to finite truncation error, the RDM yields more reliable results for the expectation value of simple operators (e.g., two-point correlation functions for short and intermediate distances) and becomes less reliable for more complex operators or at long distances. Therefore, to avoid overfitting to numerical errors, instead of considering all basis operators in the evaluation of $\Delta_{1}$, we only consider the most physically relevant operators, 
i.e., simple operators motivated by symmetry considerations, etc. For example, for the Hubbard model, we consider the following components first:

$$
\begin{aligned}
G_{A, \mathrm{t}}(i, j) & =\sum_{\sigma}\left\langle c_{i, \sigma}^{\dagger} c_{j, \sigma}\right\rangle, \quad G_{A, \mu}(i)=\left\langle n_{i}\right\rangle, \\
G_{A, \mathrm{U}}(i) & =\left\langle n_{i, \uparrow} n_{i, \downarrow}\right\rangle, \quad G_{A, \mathrm{~J}}(i, j)=\left\langle\mathbf{S}_{i} . \mathbf{S}_{j}\right\rangle, \\
G_{A, \mathrm{~V}}(i, j) & =\left\langle n_{i} n_{j}\right\rangle,
\end{aligned}
$$

and similarly for $\tilde{G}$. We then evaluate $\eta_{a}=\operatorname{Tr}\left(G_{A, a}-\widetilde{G_{A, a}}\right)^{2}$, and by combining them,

$$
\Delta_{1}=w_{t} \eta_{t}+w_{\mu} \eta_{\mu}+w_{U} \eta_{U}+w_{J} \eta_{J}+w_{V} \eta_{V} .
$$

The exact values of $w_{t}, w_{\mu}, \ldots$ are not crucial as long as they all have the same order of magnitude. Nonetheless, in most computations, we choose $w_{t}=w_{U}=w_{\mu}=w_{J}=$ $w_{V}=1$ for the weights.

(ii) Quantum relative entropy $(Q R E)$ of the two reduced density matrices: $\Delta_{2}=\operatorname{Tr}\left(\rho_{A} \ln \rho_{A}-\rho_{A} \ln \tilde{\rho}_{A}\right)$. In this method (which is closely related to the next cost function), we try to tune the couplings such that $\tilde{\rho_{A}}$ 's matrix form becomes very close to $\rho_{A}$ 's. This cost function converges significantly fast, in both the ED and DMRG methods. In ED, where we do not have to deal with truncation errors, QRE is the superior cost function and achieves correct results. However, for the DMRG (like $\Delta_{3}$ below), it also suffers from overfitting to numerical errors, i.e., those parts of $\rho_{A}$ which will change upon increasing the bond dimension of the DMRG, $\chi$ (i.e., the number of retained basis states of the Hilbert space). It is these matrix elements which are responsible for the issues related to the expectation value or $n$-point correlation functions of complex operators explained above. When $\chi$ is large enough (e.g., when the truncation error becomes less than $10^{-10}$ ), it yields results consistent with $\Delta_{1}$ 's.

(iii) Hilbert-Schmidt distance between the two reduced density matrices: $\Delta_{3}=\operatorname{Tr}\left(\rho_{A}-\tilde{\rho}_{A}\right)^{2}$. Similar to $\Delta_{2}$, in this method we try to tune the couplings such that $\tilde{\rho_{A}}$ 's matrix form becomes very close to $\rho_{A}$ 's. This cost function is slowly converging even for the ED where no truncation is involved. Moreover, for the DMRG, similar to $\Delta_{2}$, it suffers from overfitting to numerical errors, and its results are sensitive to the bond dimension, especially for small values of $\chi$.

In Sec. 4 of this Appendix we compare the results achieved via all three cost functions for the two-leg ladder Heisenberg and Hubbard models for several bond dimensions. Our results suggest that for large bond dimensions, all three methods yield consistent outcomes. However, for relatively small bond dimensions, it is $\Delta_{1}$ which performs better and results in couplings which are more consistent with the results of larger bond dimensions.

\section{Local temperature ansatz and the initial guess for couplings}

Now, let us assume that we study the following Hamiltonian:

$$
H=\sum_{\alpha} J_{\alpha} \hat{O}_{\alpha}
$$

where, due to the locality of the Hamiltonian, only certain $J_{\alpha}$ 's are nonzero. We are interested in finding the second

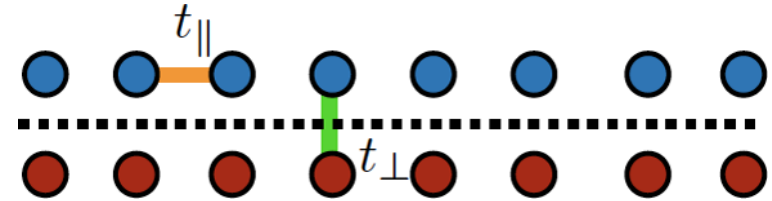

FIG. 8. We study the Hubbard model on this ladder for $U=4$, $t_{\perp}=0.5$, and $t_{\|}=1$ at half filling $(\mu=0)$. Subsystem $A$, whose $\mathrm{EH}$ is desired, is denoted by blue sites.

quantization form of the EH expanded as follows:

$$
K_{A}=\sum_{\alpha \in A} g_{\alpha} \hat{O}_{\alpha}
$$

Here, due to the renormalization procedure involved in tracing the degrees of freedom outside $A, g_{\alpha}$ 's can be viewed as our running coupling constants which $J_{\alpha}$ has flown to. Thus, in principle, any $g_{\alpha}$ consistent with symmetry considerations emerge. In practice, only a small set of them will be relevant and non-negligible.

In our algorithm, we are trying to find $g_{\alpha}$ numerically, assuming that (a subset of relevant) correlation functions are known. In our optimization algorithm, we initialized the coupling constants of the EH, $g_{\alpha}$, using the LTA's ideal form. In the LTA, the EH is local, and its coupling constants, $g_{\alpha}$ 's, are nonzero only when the corresponding couplings of the Hamiltonian (UV theory), $J_{\alpha}$ 's, are nonzero. Another task in the LTA is to assign a position to each operator. For simple two-point

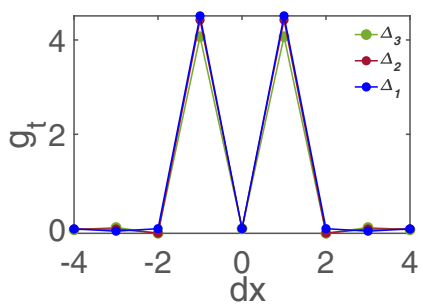

(a)

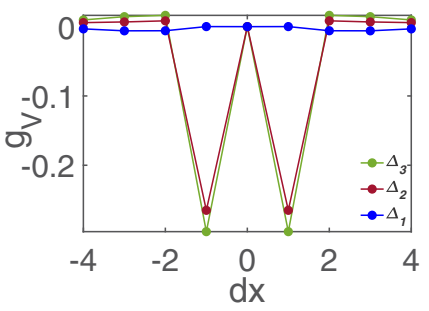

(c)

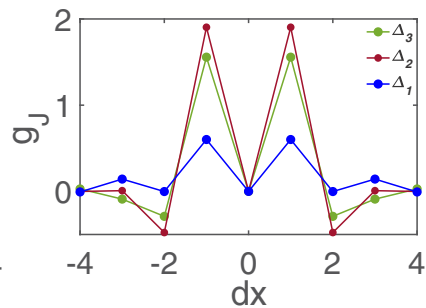

(b)

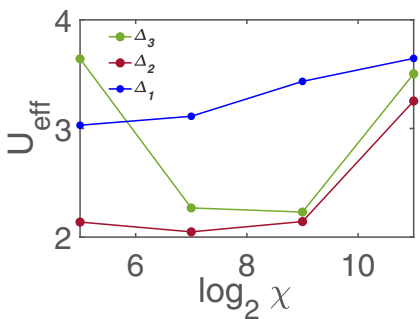

(d)
FIG. 9. $K_{A}$ 's couplings, for the geometry illustrated in Fig. 8, obtained via applying different cost functions and for various bond dimensions. Couplings are translational invariant due to the subsystem geometry. (a)-(c) $g_{t, d x}, g_{J, d x}$, and $g_{V, d x}$ achieved via GF distance $\left(\Delta_{1}\right)$, QRE distance $\left(\Delta_{2}\right)$, and RDM distance $\left(\Delta_{3}\right)$ for $\chi=2^{11}$. Also, the corresponding renormalized on-site couplings $g_{U, 0}$ are 16.4, 14.4, and 14.3 for $\Delta_{1}, \Delta_{2}$, and $\Delta_{3}$, respectively. See the text for their definitions. (d) The variation of $U_{\text {eff }}:=\frac{g_{t, 1}}{g_{U, 0}}$ vs $\log _{2} \chi$. As this plot clearly indicates, $\Delta_{1}$ is the most reliable cost function for the DMRG and exhibits the least variation. 


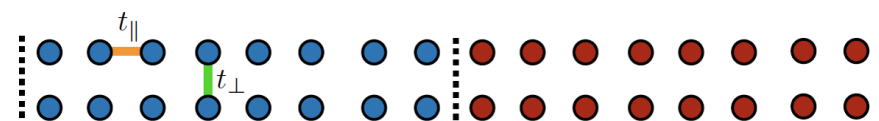

FIG. 10. We study the Hubbard model on this ladder for $U=4$, $t_{\perp}=2$, and $t_{\|}=1$ at half filling $(\mu=0)$. Subsystem $A$, whose EH is desired, is denoted by blue sites.

operators (such as $\mathbf{S}_{\mathbf{i}} \cdot \mathbf{S}_{\mathbf{j}}$ in the Heisenberg model or $c_{\mathbf{i}, \sigma}^{\dagger} c_{\mathbf{j}, \sigma}$ in the Hubbard model), the position is defined as the average position of its components, namely, $\overline{\mathbf{j}} \mathbf{j}=\frac{\mathbf{i}+\mathbf{j}}{2}$. Next, we must compute the minimum distance (geodesics) between $\overline{\mathbf{i j}}$ and the boundary separating $A$ and its environment, $B$. Let us call this minimum distance $x_{\mathrm{ij}}$. Finally, at zero temperature (for ground states) and for the open boundary condition (OBC), the LTA attributes the following form to $g_{\alpha}\left(x_{\mathrm{ij}}\right)$ [23]:

$$
g_{\alpha}\left(x_{\mathrm{ij}}\right)=J_{\alpha} \frac{4 \ell}{v} \sin \left(\frac{\pi}{2 \ell} x_{\mathrm{ij}}\right),
$$

where $\ell$ is the maximum value of $x_{\mathrm{ij}}$ (i.e., the linear dimension of $A$ normal to $\partial A$ ) and $v$ is the group velocity of low-energy excitations (quasiparticles) and is model dependent. In our algorithm, besides $v$, we also treated $\ell$ as a variational parameter. We first optimized and tuned $v$ and $\ell$. Then, we took the optimized form of local $g_{\alpha}$ (associated with $v^{*}$ and $\ell^{*}$ ), and using the gradient descent algorithm, we optimized our cost function. We allowed all relevant couplings, including

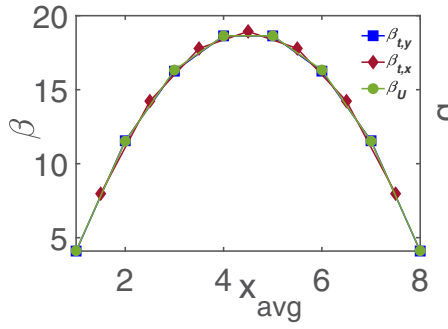

(a)

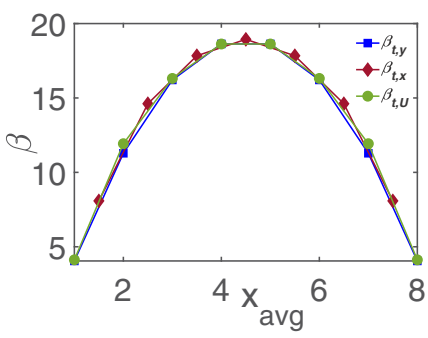

(c)

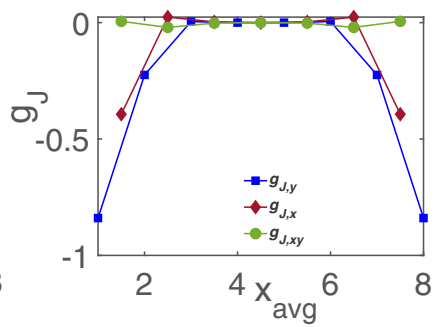

(b)

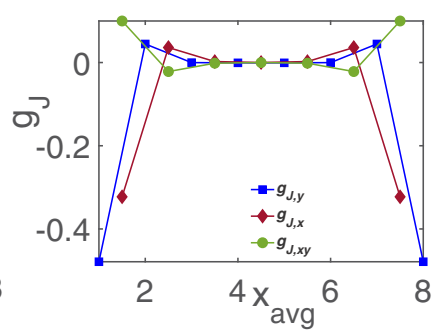

(d)
FIG. 11. $K_{A}$ 's couplings, for the geometry illustrated in Fig. 10, obtained via applying GF distance $\left(\Delta_{1}\right.$ cost function) and for $\chi=2^{11}$ and $\chi=2^{9}$. (a) Various $\beta$ profiles for $\Delta_{1}$ cost function for $\chi=2^{11}$ (see the main text for their definitions). (b) Most significant corrections to LTA corrections, $g_{J}$ for $\Delta_{1}$ cost function for $\chi=2^{11}$. The second and third neighbors' corrections to $g_{t}$ are negligible due to the particle-hole symmetry. Moreover, we found $g_{V}$ to be irrelevant as well, and that is why they are absent in this and the following two figures. (c) and (d) Same as (a) and (b) but for $\chi=2^{9}$.

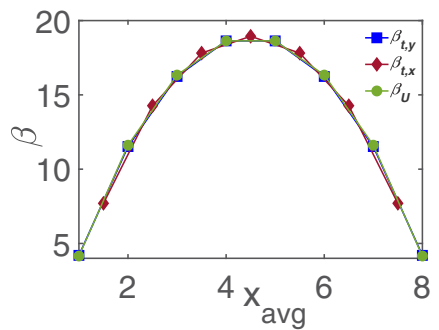

(a)

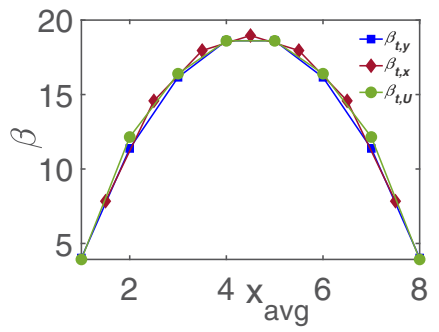

(c)

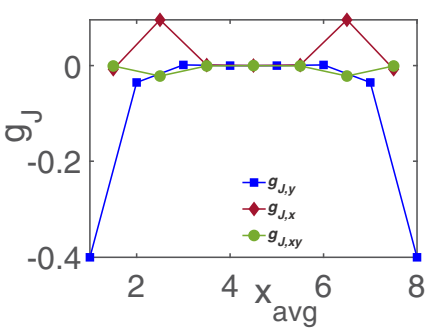

(b)

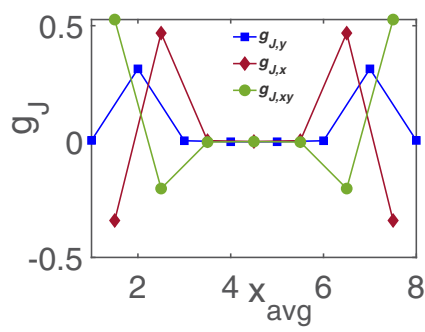

(d)
FIG. 12. Same as Fig. 11 but for the $\Delta_{2}$ cost function (QRE). Likewise, (a) and (b) are achieved by considering $\chi=2^{11}$, while (c) and (d) are achieved by considering $\chi=2^{9}$.

distant neighbors and nonlocal terms (which were absent in the system's Hamiltonian) as well as the initialized local terms, to vary and deviate from their initial point. Therefore we have not imposed locality in our procedure, although it finally emerged naturally as the optimum solution (except at the boundary of $A$ with $B$, where farther neighbors became more pronounced).

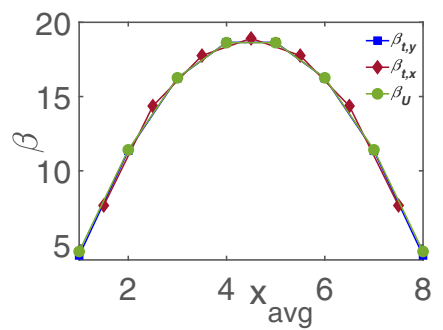

(a)

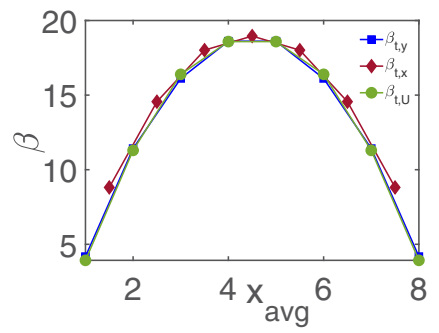

(c)

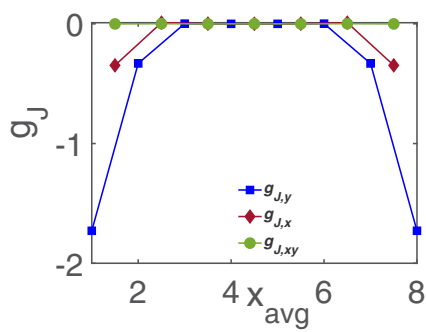

(b)

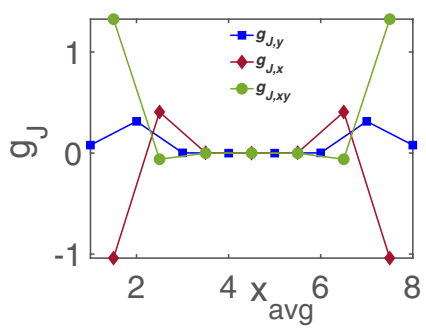

(d)
FIG. 13. Same as Fig. 11 but for the $\Delta_{3}$ cost function (RDM distance). Likewise, (a) and (b) are achieved by considering $\chi=2^{11}$, while (c) and (d) are achieved by considering $\chi=2^{9}$. 


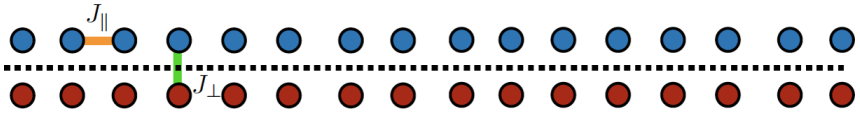

FIG. 14. We study the Heisenberg model on this ladder for $J_{\perp}=$ 0.5 and $J_{\|}=1$. Subsystem $A$, whose EH is desired, is denoted by blue sites.

Similarly, for the periodic boundary condition (PBC) at $T=0$, the LTA assigns the following value to $g_{\alpha}\left(x_{\mathrm{ij}}\right)$ [23]:

$$
g_{\alpha}\left(x_{\mathbf{i j}}\right)=J_{\alpha} \frac{2 L}{v} \frac{\sin \left(\frac{\pi}{L} x_{\mathbf{i j}}\right) \sin \left[\frac{\pi}{L}\left(\ell-x_{\mathbf{i j}}\right)\right]}{\sin \left(\frac{\pi}{L} \ell\right)},
$$

where $L$ is size of the entire system $M$ in the direction normal to $\partial A$.

\section{A detailed comparison between the performance of $\Delta_{1}, \Delta_{2}$, and $\Delta_{3}$ cost functions}

Here, we compare the EH's coefficients obtained by utilizing all three cost functions for the ladder geometry and for the Hubbard and Heisenberg models.

We first consider the undoped Hubbard model on the ladder geometry (in which $U=4, t_{\perp}=0.5, t_{\|}=1$ ) depicted in Fig. 8. This geometry results in a highly entangled subsystem: indeed, a volume law entanglement entropy. We apply all three cost functions to this system for the following five different bond dimensions: $\chi=2^{5}, 2^{7}, 2^{9}, 2^{11}$. The EH for this case is translationally invariant, namely, $g_{\tau, i_{x}, j_{x}}=g_{\tau, d x_{i j}}$ $(\tau=t, U, J, V)$, where $d x_{i j}:=j_{x}-i_{x}$. In this section, the translational symmetry is imposed on the couplings explicitly. We first compare the EH's couplings achieved via applying the GF distance $\left(\Delta_{1}\right), \operatorname{QRE}\left(\Delta_{2}\right)$, and the RDM distance $\left(\Delta_{3}\right)$ for $\chi=2^{11}$ (see Fig. 9). With this bond dimension, we can nearly probe the ground-state properties. The coefficients of local terms in the EH are almost consistent in these three methods. On the other hand, we know that ideally the ground state must exhibit particle-hole symmetry. Although $\chi=2^{11}$ is still insufficient for true convergence in the DMRG for such a highly entangled state ( $\chi=2^{12}$ seems to be enough), $\Delta_{1}$ 's

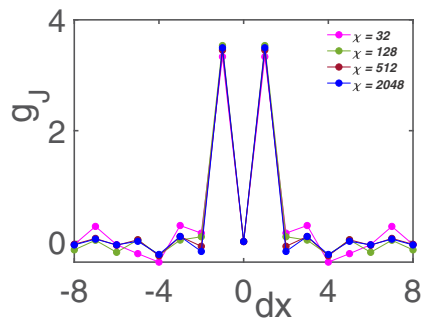

(a)

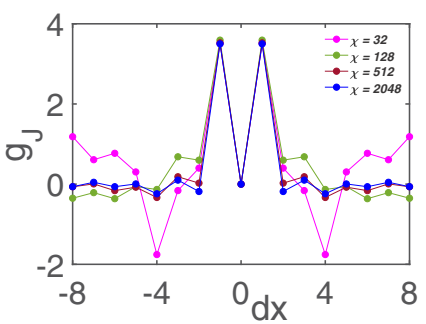

(b)
FIG. 15. $K_{A}$ 's couplings, for the geometry illustrated in Fig. 14, obtained via applying $\Delta_{1}$ and $\Delta_{2}$ cost functions and for $\chi=$ $2^{5}, 2^{7}, 2^{9}, 2^{11}$. The couplings are translationally invariant due to the geometry of $A$. (a) $g_{J, d x}$ obtained by minimizing the $\Delta_{1}$ cost function. (b) $g_{J, d x}$ obtained by minimizing the $\Delta_{2}$ cost function. As we see, both cost functions result in the same couplings for $\chi=2^{11}$. Also, the $\Delta_{1}$ results exhibit much less fluctuation than $\Delta_{2}$ 's and thus are more reliable for smaller bond dimensions ( $\chi$ 's).

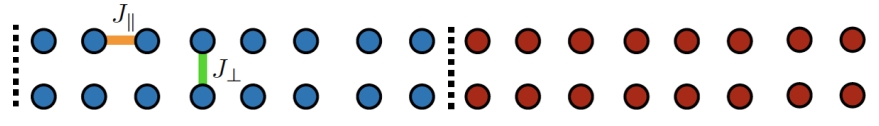

FIG. 16. We study the Heisenberg model on this ladder for $J_{\perp}=$ 2 and $J_{\|}=1$. Subsystem $A$, whose EH is desired, is denoted by blue sites.

results reflect the particle-hole symmetry [e.g., the (renormalized) second-neighbor hoppings are infinitesimal], while for those of $\Delta_{2}$ and $\Delta_{3}$ the particle-hole symmetry is visibly violated due to the overfitting issues mentioned previously. Furthermore, a previous quantum-Monte-Carlo-based study of a similar situation [32] indicated the irrelevance of $g_{V}$ couplings, which is consistent with $\Delta_{1}$ estimations. Additionally, perturbative studies of the $\mathrm{EH}$ indicate oscillating spin-spin couplings [39-41] (though subdominant to the renormalized on-site interaction), which agrees well with our results via minimizing GF distance $\left(\Delta_{1}\right)$, while those of $\Delta_{2}$ and $\Delta_{3}$ exhibit deviations in addition to their overestimation for the spin-spin couplings. In Fig. 9(d), we plot the normalized on-site interaction strength $U_{\text {eff }}^{(\chi)}:=\frac{g_{U}^{(x)}}{g_{t}^{(x)}(1)}$ for all four bond dimensions considered in our investigations. Again, as we see in Fig. 9(d), the results of the GF distance $\left(\Delta_{1}\right)$ are more robust and less sensitive to $\chi$, despite several orders of magnitude change in $\chi$, while those of the QRE and RDM distance display stronger fluctuations.

Now, we turn to the geometry shown in Fig. 10 (where $U=4, t_{\perp}=2, t_{\|}=1$ ) and present our results for all three cost functions in Figs. 11, 12, and 13. Here, we have defined

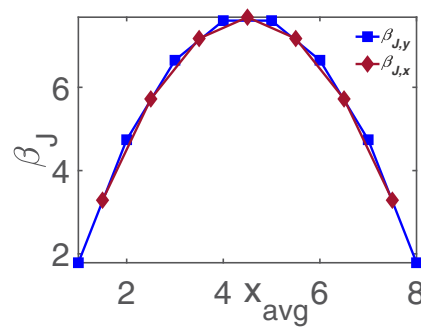

(a)

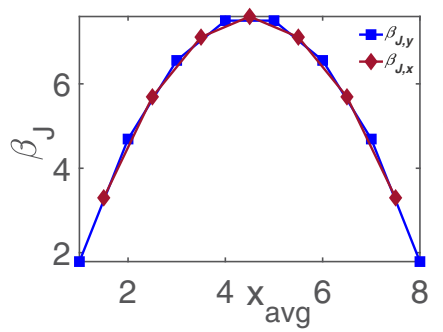

(c)

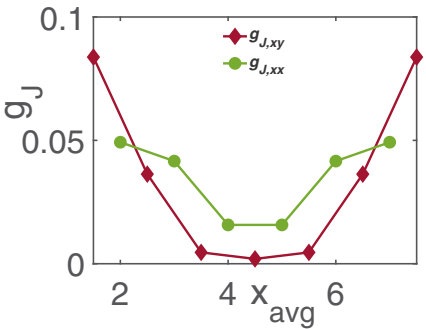

(b)

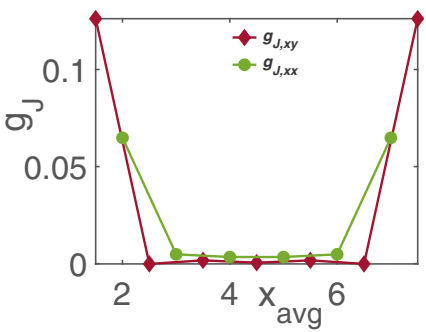

(d)
FIG. 17. $K_{A}$ 's couplings, for the geometry illustrated in Fig. 14, obtained via applying the $\Delta_{1}$ cost function and for $\chi=2^{7}, 2^{11}$. (a) and (c) show the inverse local temperatures $\left[\beta_{y}\left(i_{x}\right):=\frac{1}{J_{\perp}} g_{J, i_{x}, i_{x}}, \beta_{x}\left(i_{x}+1 / 2\right):=\frac{1}{J_{\|}} g_{J, i_{x}, i_{x}+1}\right]$ for $\chi=2^{11}$ and $2^{7}$, respectively. (b) and (d) present the second- and third-neighbor couplings of the $\mathrm{EH}$. 


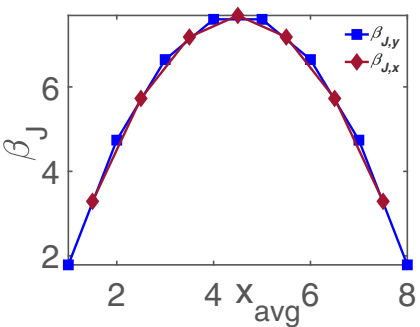

(a)

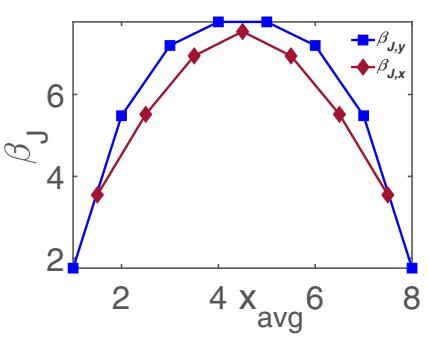

(c)

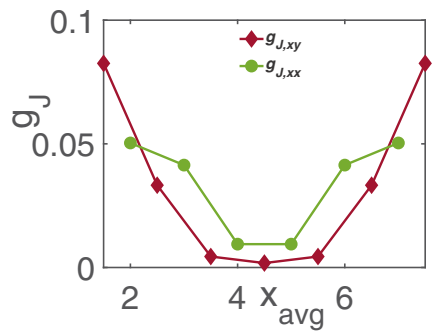

(b)

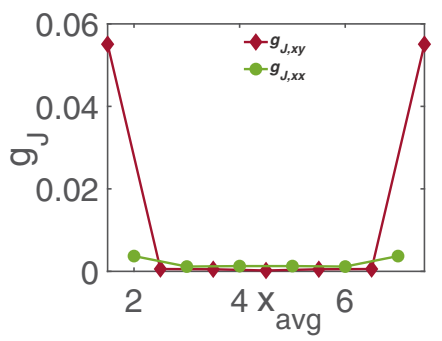

(d)
FIG. 18. Same as Fig. 17 but for the $\Delta_{2}$ cost function. (a) and (b) are obtained by keeping $\chi=2^{11}$ basis states of the Hilbert space in the DMRG, while (c) and (d) are obtained by keeping $\chi=2^{7}$ basis states.

the following inverse local temperatures: $\beta_{t, x}\left(i_{x}+1 / 2\right):=$ $g_{t, \mathbf{i}, \mathbf{i}+\hat{\mathbf{x}}}, \beta_{t, y}\left(i_{x}\right):=g_{t, \mathbf{i}, \mathbf{i}+\hat{\mathbf{y}}}$ and $\beta_{U}\left(i_{x}\right):=\frac{1}{U} g_{U, \mathbf{i}}$. For this problem, due to the PBC imposed along the $x$ direction, we found that even with $\chi=2^{11}$, there is still some room for the DMRG to converge to the true ground state. As a result, we still see some minor discrepancies among the results of the three methods for $\chi=2^{11}$ for subdominant and correction terms beyond the LTA (though they yield highly correlated results). Nonetheless, the local terms (i.e., dominant couplings) are reasonably consistent. We have also plotted the results of $\chi=2^{9}$ for all three methods, and again, $\Delta_{1}$ 's results proved to be more robust and $\Delta_{2}$ and $\Delta_{3}$ 's less stable. Thus, in the presence of truncation errors, we can trust the results of the GF distance more than those of the other two candidates for the cost function.

For the sake of completeness, we have also explored the robustness and the accuracy of the above three cost function candidates for the Heisenberg model, again on a ladder geometry. To this end, we first studied the geometry shown in Fig. 14 (where $J_{\perp}=0.5$ and $J_{\|}=1$ ) and presented its results in Fig. 15. Similar to the Hubbard model case, this geometry leads to a highly entangled ground state. Likewise, we expect translationally invariant couplings, namely, $g_{J, i_{x}, j_{x}}=g_{J, d x_{i j}}$. We have presented $g_{J, d x}$ for $\chi=2^{5}, 2^{7}, 2^{9}, 2^{11}$ for the GF and QRE cost functions (the RDM cost function yields results similar to those of the QRE). In this case as well, the GF distance turns out to be the most stable one.

Finally, we studied the Heisenberg model on the geometry illustrated in Fig. 16 (in which $J_{\perp}=0.5$ and $J_{\|}=1$ ). The results are presented in Figs. 17 and 18 for the GF distance and QRE, respectively. For this problem, we indeed achieved the true ground state using $\chi=2^{11}$. Therefore all cost functions must achieve the same couplings. On the other hand, for smaller bond dimensions, e.g., $\chi=2^{7}, \Delta_{1}$ achieves more accurate results (relative to $\chi=2^{11}$ ) than the remaining cost functions.
[1] L. Susskind and J. Lindesay, An Introduction to Black Holes, Information and the String Theory Revolution: The Holographic Universe (World Scientific, Hackensack, 2005).

[2] M. Nielsen and I. Chuang, Quantum Computation and Quantum Information (Cambridge University Press, Cambridge, 2000).

[3] S. Ryu and T. Takayanagi, Holographic Derivation of Entanglement Entropy from the anti-de Sitter Space/Conformal Field Theory Correspondence, Phys. Rev. Lett. 96, 181602 (2006).

[4] A. Kitaev and J. Preskill, Topological Entanglement Entropy, Phys. Rev. Lett. 96, 110404 (2006).

[5] M. Levin and X.-G. Wen, Detecting Topological Order in a Ground State Wave Function, Phys. Rev. Lett. 96, 110405 (2006).

[6] H. Li and F. D. M. Haldane, Entanglement Spectrum as a Generalization of Entanglement Entropy: Identification of Topological Order in Non-Abelian Fractional Quantum Hall Effect States, Phys. Rev. Lett. 101, 010504 (2008).

[7] A. Sterdyniak, A. Chandran, N. Regnault, B. A. Bernevig, and P. Bonderson, Real-space entanglement spectrum of quantum Hall states, Phys. Rev. B 85, 125308 (2012).

[8] R. Thomale, A. Sterdyniak, N. Regnault, and B. A. Bernevig, Entanglement Gap and a New Principle of Adiabatic Continuity, Phys. Rev. Lett. 104, 180502 (2010).
[9] A. M. Läuchli, E. J. Bergholtz, J. Suorsa, and M. Haque, Disentangling Entanglement Spectra of Fractional Quantum Hall States on Torus Geometries, Phys. Rev. Lett. 104, 156404 (2010).

[10] Z. Liu, A. Vaezi, K. Lee, and E.-A. Kim, Non-Abelian phases in two-component $v=2 / 3$ fractional quantum Hall states: Emergence of Fibonacci anyons, Phys. Rev. B 92, 081102(R) (2015).

[11] Z.-P. Cian, T. Grass, A. Vaezi, Z. Liu, and M. Hafezi, Engineering quantum Hall phases in a synthetic bilayer graphene system, Phys. Rev. B 102, 085430 (2020).

[12] M.-S. Vaezi and A. Vaezi, Numerical observation of parafermion zero modes and their stability in 2D topological states, arXiv:1706.01192 [cond-mat.str-el].

[13] M. P. Zaletel, R. S. K. Mong, and F. Pollmann, Topological Characterization of Fractional Quantum Hall Ground States from Microscopic Hamiltonians, Phys. Rev. Lett. 110, 236801 (2013).

[14] X.-L. Qi, H. Katsura, and A. W. W. Ludwig, General Relationship between the Entanglement Spectrum and the Edge State Spectrum of Topological Quantum States, Phys. Rev. Lett. 108, 196402 (2012).

[15] F. Pollmann, A. M. Turner, E. Berg, and M. Oshikawa, Entanglement spectrum of a topological phase in one dimension, Phys. Rev. B 81, 064439 (2010). 
[16] H. Yarloo, A. Langari, and A. Vaezi, Anyonic self-induced disorder in a stabilizer code: Quasi many-body localization in a translational invariant model, Phys. Rev. B 97, 054304 (2018).

[17] P. Calabrese and J. Cardy, Entanglement entropy and conformal field theory, J. Phys. A: Math. Theor. 42, 504005 (2009).

[18] H. Pichler, G. Zhu, A. Seif, P. Zoller, and M. Hafezi, Measurement Protocol for the Entanglement Spectrum of Cold Atoms, Phys. Rev. X 6, 041033 (2016).

[19] L. D'Alessio, Y. Kafri, A. Polkovnikov, and M. Rigol, From quantum chaos and eigenstate thermalization to statistical mechanics and thermodynamics, Adv. Phys. 65, 239 (2016).

[20] J. J. Bisognano and E. H. Wichmann, On the duality condition for a Hermitian scalar field, J. Math. Phys. (Melville, NY) 16, 985 (1975).

[21] J. J. Bisognano and E. H. Wichmann, On the duality condition for quantum fields, J. Math. Phys. (Melville, NY) 17, 303 (1976).

[22] H. Casini, M. Huerta, and R. C. Myers, Towards a derivation of holographic entanglement entropy, J. High Energy Phys. 05 (2011) 036.

[23] J. Cardy and E. Tonni, Entanglement Hamiltonians in twodimensional conformal field theory, J. Stat. Mech. (2016) 123103.

[24] R. E. Arias, D. D. Blanco, H. Casini, and M. Huerta, Local temperatures and local terms in modular Hamiltonians, Phys. Rev. D 95, 065005 (2017).

[25] R. Bousso, H. Casini, Z. Fisher, and J. Maldacena, Entropy on a null surface for interacting quantum field theories and the Bousso bound, Phys. Rev. D 91, 084030 (2015).

[26] S. M. S. Vaezi, Z. Nussinov, and A. Vaezi, Local temperature approximation of entanglement Hamiltonian, Bull. Am. Phys. Soc. 63, Y20.00010 (2018).

[27] A. Vaezi, Local temperature ansatz: A novel quantum entanglement based approach for solving strongly correlated systems, lecture delivered at Georgia Institute of Technology, Atlanta, 2018, https://smartech.gatech.edu/handle/1853/59339.

[28] X. Turkeshi, T. Mendes-Santos, G. Giudici, and M. Dalmonte, Entanglement-Guided Search for Parent Hamiltonians, Phys. Rev. Lett. 122, 150606 (2019).

[29] M.-C. Chung and I. Peschel, Density-matrix spectra of solvable fermionic systems, Phys. Rev. B 64, 064412 (2001).

[30] S.-A. Cheong and C. L. Henley, Many-body density matrices for free fermions, Phys. Rev. B 69, 075111 (2004).

[31] I. Peschel and V. Eisler, Reduced density matrices and entanglement entropy in free lattice models, J. Phys. A: Math. Theor. 42, 504003 (2009).

[32] F. P. Toldin and F. F. Assaad, Entanglement Hamiltonian of Interacting Fermionic Models, Phys. Rev. Lett. 121, 200602 (2018).

[33] M. Dalmonte, B. Vermersch, and P. Zoller, Quantum simulation and spectroscopy of entanglement Hamiltonians, Nat. Phys. 14, 827 (2018).

[34] G. Giudici, T. Mendes-Santos, P. Calabrese, and M. Dalmonte, Entanglement Hamiltonians of lattice models via the Bisognano-Wichmann theorem, Phys. Rev. B 98, 134403 (2018).

[35] V. Eisler, E. Tonni, and I. Peschel, On the continuum limit of the entanglement Hamiltonian, J. Stat. Mech. (2019) 073101.
[36] T. Mendes-Santos, G. Giudici, M. Dalmonte, and M. A Rajabpour, Entanglement Hamiltonian of quantum critical chains and conformal field theories, Phys. Rev. B 100, 155122 (2019).

[37] G. Wong, I. Klich, L. A. P. Zayas, and D. Vaman, Entanglement temperature and entanglement entropy of excited states, J. High Energy Phys. 2013 (2013) 732682.

[38] W. Zhu, Z. Huang, and Y.-C. He, Reconstructing entanglement Hamiltonian via entanglement eigenstates, Phys. Rev. B 99, 235109 (2019).

[39] S. Furukawa and Y. B. Kim, Erratum: Entanglement entropy between two coupled Tomonaga-Luttinger liquids [Phys. Rev. B 83, 085112 (2011)], Phys. Rev. B 87, 119901(E) (2013).

[40] A. M. Läuchli and J. Schliemann, Entanglement spectra of coupled $s=\frac{1}{2}$ spin chains in a ladder geometry, Phys. Rev. B 85, 054403 (2012).

[41] X. Chen and E. Fradkin, Quantum entanglement and thermal reduced density matrices in fermion and spin systems on ladders, J. Stat. Mech. (2013) P08013.

[42] X. Wen, S. Ryu, and A. W. W. Ludwig, Entanglement Hamiltonian evolution during thermalization in conformal field theory, J. Stat. Mech. (2018) 113103.

[43] J. Koeller, S. Leichenauer, A. Levine, and A. ShahbaziMoghaddam, Local modular Hamiltonians from the quantum null energy condition, Phys. Rev. D 97, 065011 (2018).

[44] N. Lashkari, Modular Hamiltonian for Excited States in Conformal Field Theory, Phys. Rev. Lett. 117, 041601 (2016).

[45] G. D. Giulio and E. Tonni, On entanglement Hamiltonians of an interval in massless harmonic chains, J. Stat. Mech. (2020) 033102.

[46] J. Zhang, P. Calabrese, M. Dalmonte, and M. A. Rajabpour, Lattice Bisognano-Wichmann modular Hamiltonian in critical quantum spin chains, SciPost Phys. Core 2, 007 (2020).

[47] W. Zhu, Z. Huang, Y.-C. He, and X. Wen, Entanglement Hamiltonian of Many-Body Dynamics in Strongly Correlated Systems, Phys. Rev. Lett. 124, 100605 (2020).

[48] C. Kokail, R. van Bijnen, A. Elben, B. Vermersch, and P. Zoller, Entanglement Hamiltonian tomography in quantum simulation, arXiv:2009.09000 [quant-ph].

[49] S. R. White, Density Matrix Formulation for Quantum Renormalization Groups, Phys. Rev. Lett. 69, 2863 (1992).

[50] B. Swingle and I. H. Kim, Reconstructing Quantum States from Local Data, Phys. Rev. Lett. 113, 260501 (2014).

[51] A. W. Sandvik, Finite-size scaling of the ground-state parameters of the two-dimensional Heisenberg model, Phys. Rev. B 56, 11678 (1997).

[52] E. M. Stoudenmire and S. R. White, Studying two-dimensional systems with the density matrix renormalization group, Annu. Rev. Condens. Matter Phys. 3, 111 (2012).

[53] H.-C. Jiang, H. Yao, and L. Balents, Spin liquid ground state of the spin- $\frac{1}{2}$ square $J_{1}-J_{2}$ Heisenberg model, Phys. Rev. B 86, 024424 (2012).

[54] C. N. Varney, C.-R. Lee, Z. J. Bai, S. Chiesa, M. Jarrell, and R. T. Scalettar, Quantum Monte Carlo study of the twodimensional fermion Hubbard model, Phys. Rev. B 80, 075116 (2009).

[55] F. Šimkovic, J. P. F. LeBlanc, A. J. Kim, Y. Deng, N. V. Prokof'ev, B. V. Svistunov, and E. Kozik, Extended Crossover from a Fermi Liquid to a Quasiantiferromagnet in the 
Half-Filled 2D Hubbard Model, Phys. Rev. Lett. 124, 017003 (2020).

[56] Y.-F. Jiang, J. Zaanen, T. P. Devereaux, and H.-C. Jiang, Ground state phase diagram of the doped Hubbard model on the four-leg cylinder, Phys. Rev. Res. 2, 033073 (2020).

[57] E. W. Huang, C. B. Mendl, S. Liu, S. Johnston, H.-C. Jiang, B. Moritz, and T. P. Devereaux, Numerical evidence of fluctuating stripes in the normal state of high- $T_{\mathrm{c}}$ cuprate superconductors, Science 358, 1161 (2017).

[58] E. Fradkin, S. A. Kivelson, and J. M. Tranquada, Colloquium: Theory of intertwined orders in high temperature superconductors, Rev. Mod. Phys. 87, 457 (2015).

[59] Y. Zhou, K. Kanoda, and T.-K. Ng, Quantum spin liquid states, Rev. Mod. Phys. 89, 025003 (2017). 